\title{
EFEITO DA EXTRUSÃO TERMOPLÁSTICA NAS CARACTERÍSTICAS DE VISCOSIDADE DE PASTA, SOLUBILIDADE E ABSORÇÃO DE ÁGUA DE FARINHAS PRÉ-GELATINIZADAS DE MILHO E FEIJÃO CARIOCA (BRS PONTAL)
}

\author{
ERIKA MADEIRA MOREIRA DA SILVA* \\ JOSÉ LUIS RAMÍREZ ASCHERI** \\ DIEGO PALMIRO RAMÍREZ ASCHERI ${ }^{* * *}$ \\ CARLA DA SILVA TEBA ${ }^{* * * *}$
}

\begin{abstract}
O objetivo deste trabalho foi avaliar o efeito da extrusão termoplástica nas características de pasta, solubilidade e absorção de água de farinhas pré-gelatinizadas de milho e feijão do tipo carioca (BRS Pontal). Os parâmetros utilizados no processamento por extrusão termoplástica foram: rotação do parafuso (318,2 a 392,9 rpm); umidade inicial na mistura (10,9 a 21\%) e adição de feijão sem casca BRS Pontal do tipo carioca (4,8 a 55,2\%). Esses parâmetros foram estabelecidos, nos seus devidos níveis, por meio de delineamento experimental composto rotacional de segunda ordem, cujas variáveis respostas estudadas foram: índice de solubilidade em água (ISA), índice de absorção de água (IAA), viscosidade máxima inicial (V25), viscosidade máxima a $95^{\circ} \mathrm{C}$ (VMax) e viscosidade final (VF). Por meio do estudo realizado verificou-se que o aumento do conteúdo de feijão nas amostras elevou a solubilidade das farinhas extrudadas, entretanto não influenciou de forma significativa os valores de absorção em água. A viscosidade de pasta apresentada pelas farinhas está condizente com a característica de prégelatinizada, sendo solubilizadas sem a necessidade de cozimento. Os pontos ótimos considerados para a elaboração das farinhas prégelatinizadas foram: ISA, 13,07 \%; IAA, $5,81 \mathrm{~g}$ gel/g matéria seca; V25, 436,52 cP; VMax, 157,86 cP e VF, 390,26 cP para valor de desejabilidade de 0,90 .
\end{abstract}

PALAVRAS-CHAVE: EXTRUSÃO; FARINHAS PRÉ-GELATINIZADAS; MILHO; FEIJÃO.

* Doutora em Ciência e Tecnologia de Alimentos, Departamento de Educação Integrada em Saúde, Centro de Ciências da Saúde, Universidade Federal do Espírito Santo, Vitória, ES (e-mail: erika.alimentos@ gmail.com).

** Doutor em Tecnologia de Alimentos, Embrapa Agroindústria de Alimentos, Rio de Janeiro, RJ (e-mail: ascheri@ctaa.embrapa.br).

*** Doutor em Engenharia de Alimentos, Unidade de Ciências Exatas e Tecnológicas, Universidade Estadual de Goiás, Anápolis, GO (e-mail: ascheridpr@gmail.com).

**** Doutoranda em Ciência e Tecnologia de Alimentos, Universidade Federal Rural do Rio de Janeiro, Rio de Janeiro, RJ (e-mail: carla.teba@gmail.com). 


\section{INTRODUÇÃO}

Cada vez mais os consumidores têm optado por produtos que tenham conveniência na preparação, tornando necessário que a indústria produza alimentos prontos ou semi-prontos e que ao mesmo tempo sejam equilibrados nutricionalmente. Essa tendência pode ser alcançada com a utilização da extrusão para a elaboração de novos produtos. Por meio dessa tecnologia, variandose alguns parâmetros de processamento, podem ser obtidas farinhas pré-gelatinizadas a partir da mistura de matérias-primas nutricionalmente complementares e equilibradas.

Farinhas e amidos pré-gelatinizados podem ser usados sem emprego do calor (por exemplo em pudins, misturas lácteas instantâneas e alimentos para desjejum) para preparar misturas prontas panificáveis, cujo aumento da absorção e retenção de água melhora a qualidade do produto (CLERICl e EL-DASH, 2008). A pré-gelatinização de farinhas pode ser obtida em escala industrial pela utilização da extrusão. Essa se apresenta como um dos mais vantajosos métodos para substituir o processo convencional na produção de farinhas pré-gelatinizadas, devido ao controle mais rigoroso do grau de gelatinização, em que pequenas modificações no equipamento e/ ou na matéria-prima podem levar a diferentes resultados finais na qualidade do produto (CLERICI e EL-DASH, 2008).

As principais propriedades funcionais dos extrudados envolvem a absorção de água, a solubilidade em água e a viscosidade (LAZOU e KROKIDA, 2010). O aumento de solubilidade do material serve como base para a elaboração de alimentos de fácil reconstituição como, por exemplo, as sopas. Após a cocção por extrusão, as farinhas de alguns cereais são comercializadas como farinhas pré-gelatinizadas.

O feijão, geralmente adquirido na forma de grãos secos e crus, demanda longo período de tempo para seu preparo, diminuindo sua competitividade em relação aos produtos semiprontos ou prontos para o consumo (GOMES et al., 2006). No entanto, o feijão apresenta em sua composição de 21 a $25 \%$ de proteínas, de 58 a $64 \%$ de carboidratos e cerca de 1,5\% de lipídios. Contém também, teor de fibras (variando de 14 a 19\%) mais elevado que os cereais. É considerado alimento com alto valor nutricional por ser composto de vitaminas do complexo $\mathrm{B}$ e minerais (tais como, ferro, magnésio, zinco e fósforo), além de fornecer cálcio, cobre, manganês, potássio e sódio (MESQUITA, CORRÊA e ABREU, 2007). Com o lançamento de diversas sopas desidratadas à base de feijão, 0 consumo de farinha desse grão aumentou expressivamente (GOMES e SILVA, 2003).

O cultivar BRS Pontal, grão do tipo Carioca com grande aceitação no mercado, está se destacando nos programas de biofortificação por apresentar maiores teores de ferro e zinco devido melhoramento convencional. Sua resistência à seca constitui fator positivo quanto à produtividade, principalmente em regiões de seca.

Apesar do milho em grão não ter participação muito grande na alimentação humana, seu consumo cresceu 15\% de 2004 até 2011, com expectativa de aumento para 1900 toneladas para o ano de 2012. A forma de consumo do milho mais relevante ocorre por meio de seus derivados, principalmente em regiões com menor desenvolvimento (MIRANDA, DUARTE e GARCIA, 2012). Nutricionalmente, a porção amilácea do grão (endosperma) constitui-se de amido e traços de minerais e gordura. Já o tegumento e a ponta são constituídos de carboidratos, embora apresentem menos amido e cerca de $15 \%$ de fibra bruta (ANDRIGUETTO, 2006).

O objetivo deste trabalho foi avaliar o efeito da extrusão termoplástica nas características de viscosidade de pasta, solubilidade e absorção de água de farinhas pré-gelatinizadas de milho e feijão do tipo carioca (BRS Pontal).

\section{MATERIAL E MÉTODOS}

\subsection{MATÉRIA-PRIMA}

Utilizou-se como base para a elaboração das farinhas, milho comercial na forma de grits. A essa matéria-prima foram acrescidas diferentes proporções de farinha de feijão (Phaseolus 
vulgaris L.) BRS Pontal, do tipo carioca, fornecido pela Embrapa Arroz e Feijão (Goiânia). Previamente ao preparo da farinha, os grãos de feijão foram imersos em água a $40^{\circ} \mathrm{C}$ por 4 horas, drenados, secos em estufa com circulação de ar (Fabbe-Primar, São Paulo, SP, Brasil) a $100^{\circ} \mathrm{C}$ por 1 hora e descascados em descascador provido de discos de pedra. Posteriormente, os grãos foram moídos em moinho de discos da marca Perten, Laboratory Mill 3600 (Sweden), usando-se abertura no nível zero do equipamento para obter a farinha.

Os testes, assim como o desenvolvimento das farinhas pré-gelatinizadas, foram conduzidos na planta piloto de cereais da Embrapa Agroindústria de Alimentos (RJ).

\subsection{CLASSIFICAÇÃO GRANULOMÉTRICA}

A classificação granulométrica das matérias-primas seguiu a metodologia proposta por Ascheri e Carvalho (2008). Para a classificação granulométrica do grits de milho e feijão foram peneiradas $100 \mathrm{~g}$ dessas matérias-primas cruas, durante 10 minutos, em conjunto de sete peneiras arredondadas e vibratórias com aberturas das malhas de 1200, 1000, 853, 710, 422, 354, $297 \mu \mathrm{m}$ (marca RO-TAP, modelo RX-29-10). Em seguida, as quantidades retidas em cada peneira foram pesadas em balança digital da marca Mettler Toledo, modelo SB 12001, sendo os valores expressos em porcentagens.

\subsection{PROCESSO DE EXTRUSÃO DAS MISTURAS}

Ao grits de milho foram acrescidas diferentes proporções de feijão $(4,8$ a $55,2 \%)$, sendo as misturas posteriormente condicionadas para diferentes níveis de umidade (10,9a21,0\%). A quantidade de água potável a ser adicionada foi obtida de acordo com a Equação 1 (ASCHERI e CARVALHO, 2008):

$$
Y=\frac{(U f-U i) \times M a}{100-U f}
$$

Em que:

$\mathrm{Y}=$ quantidade de água a ser adicionada $(\mathrm{mL})$;

Uf = umidade final da amostra $(\%)$

$\mathrm{Ui}=$ umidade inicial da amostra $(\%) \mathrm{e}$

$\mathrm{Ma}=$ massa da amostra $(\mathrm{g})$.

As amostras, após adicionadas de água potável, foram homogeneizadas utilizando-se batedeira industrial com agitação durante 5 minutos, colocadas em sacos plásticos e armazenadas sob refrigeração durante tempo médio de 17 horas para se obter distribuição e absorção uniforme da água. Após esse período, as farinhas mistas foram retiradas do refrigerador até alcançarem temperatura ambiente e posteriormente submetidas ao processo de extrusão.

O processo de extrusão foi realizado em extrusor de rosca simples da marca Inbra RX50 (Ribeirão Preto, SP, Brasil). Manteve-se, durante todo o processo de extrusão, a taxa de alimentação na calha dosadora a $70 \%$ e a rotação da faca a $33 \mathrm{rpm}$.

Para o processo de extrusão foi utilizada matriz dupla dotada de duas partes. A posterior, com diversos orifícios, aumenta a resistência ao fluxo e distribui uniformemente a massa proveniente do canhão. A parte exterior, dotada de quatro aberturas circulares de $3 \mathrm{~mm}$ de diâmetro cada, eleva a resistência ao fluxo e formata o produto. A vazão de produção dos extrudados ficou entre $30 \mathrm{e}$ $58 \mathrm{~kg} / \mathrm{h}$ ao passo que o torque situou-se entre 52 e $90 \%$. A fim de se obter amostras homogêneas e semelhantes, os extrudados foram recolhidos em sacos plásticos após 1 minuto de processamento. Os extrudados obtidos foram dispostos em bandejas e encaminhados para estufa Fabbe-Primar (São Paulo, SP, Brasil) com circulação forçada de ar a $50^{\circ} \mathrm{C}$, durante 15 horas ou até atingirem umidade final entre 4 e $6 \%$. Para a elaboração das farinhas, os extrudados expandidos foram submetidos a moagem em moinho de discos (Perten, Laboratory Mill 3600, Sweden) com abertura de discos equivalente ao nível zero. 


\section{4 ÍNDICE DE SOLUBILIDADE EM ÁGUA (ISA) E ÍNDICE DE ABSORÇÃO DE ÁGUA (IAA)}

A determinação dos índices de solubilidade em água (ISA) e de absorção de água (IAA) das amostras foi realizada conforme os princípios básicos do método descrito por Anderson et al. (1969).

\subsection{VISCOSIDADE DE PASTA}

A viscosidade de pasta foi determinada em "Rapid Visco Analyser (RVA)", seguindo-se a metodologia proposta por Ascheri et al. (2006). Para essa análise foram utilizadas amostras com granulometria semelhante, entre 125 e $250 \mu \mathrm{m}$, conforme proposto por Becker, Hill e Mitchell (2001).

Utilizou-se o perfil de análise extrusion 1 no-alcohol, sendo analisados os parâmetros: viscosidade máxima a $25^{\circ} \mathrm{C}(\mathrm{V} 25)$, viscosidade máxima (VMax) e viscosidade final (VF) no ciclo de resfriamento. Os resultados foram expressos em centipoise (cP).

\subsection{ESTUDO EXPERIMENTAL E ANÁLISE ESTATÍSTICA}

As variáveis escolhidas para o processamento dos extrudados, conforme estudos preliminares, foram: rotação do parafuso ( $\mathrm{rpm})$, umidade do processamento (\%) e feijão (\%) na mistura com grits de milho. Para analisar o efeito combinado dessas variáveis nas características tecnológicas dos extrudados estabeleceu-se delineamento do tipo composto central rotacional de $2^{\text {a }}$ ordem (BOX, HUNTER e HUNTER, 1978) com 6 pontos axiais, 8 pontos fatoriais e 6 pontos centrais, servindo como estimativa do erro experimental para determinar a precisão do modelo matemático, totalizando 20 tratamentos. Usando a análise de variância (ANOVA), mediante teste de distribuição $F$ a $5 \%$ de probabilidade, aplicou-se equação de segunda ordem para estabelecer modelo matemático preditivo das variáveis resposta, cuja equação geral (BOX, HUNTER e HUNTER, 1978) foi:

$$
Y i=\beta_{0}+\beta_{1} X_{1}+\beta_{2} X_{2}+\beta_{3} X_{3}+\underset{11}{\beta_{23} X_{1}^{2}+\beta_{22} X_{2}{ }^{2}+\beta_{33} X_{3}^{2}+\beta_{12} X_{1} X_{2}+\beta_{13} X_{1} X_{3}+}
$$

Em que:

$Y \mathbf{i}=$ função resposta genérica; $\mathrm{X}_{1}, \mathrm{X}_{2}, \mathrm{X}_{3}=$ valores das variáveis independentes decodificadas; $\boldsymbol{\beta}_{0}=$ coeficiente relativo à interseção do plano com o eixo resposta; $\boldsymbol{\beta}_{1}, \boldsymbol{\beta}_{2}, \boldsymbol{\beta}_{3}=$ coeficientes lineares estimados pelo método dos mínimos quadrados; $\boldsymbol{\beta}_{11}, \boldsymbol{\beta}_{23}, \boldsymbol{\beta}_{33}=$ coeficientes das variáveis quadráticas; $\boldsymbol{\beta}_{12}, \boldsymbol{\beta}_{13}, \boldsymbol{\beta}_{23}=$ coeficientes de interação entre as variáveis independentes; $\boldsymbol{\xi}=$ erro experimental.

A partir da equação de regressão obtida e oscilando os valores de duas variáveis, enquanto a terceira permanecia constante, foi possível elaborar gráficos de superfície de contorno com o auxílio da função Experimental Design (DOE) (STATISTICA, 2001). Para a análise de variância dos resultados foram utilizados os valores codificados, ao passo que para a construção dos gráficos os mesmos foram decodificados. Obtido o modelo polinomial ajustado às respostas, a otimização do processo foi efetuada pela técnica proposta para variáveis dependentes (DERRINGER e SUICH, 1980). Essa se baseia na definição de uma função de desejabilidade $\left(\mathrm{d}_{\mathrm{i}}\right)$ restrita ao intervalo de $0 \leq$ $d_{i} \leq 1$, sendo $d_{i}=1$ para a resposta desejada e $d_{i}=0$ para a resposta além da região aceitável do valor desejado. As variáveis independentes foram escolhidas depois de maximizada a desejabilidade global (D):

Em que:

$$
D=\left(d_{1} d_{2} \ldots d_{m}\right)^{1 / m}
$$

$\mathrm{m}$ = número de variáveis de resposta.

As análises estatísticas e o processamento de todos os dados foram efetuados com o auxílio do programa computacional Statistica, versão 6.0 (STATISTICA, 2001). 


\section{RESULTADOS E DISCUSSÃO}

\subsection{CLASSIFICAÇÃO GRANULOMÉTRICA DAS MATÉRIAS-PRIMAS}

A distribuição do tamanho de partículas está intimamente relacionada ao processo de moagem das matérias-primas. O feijão, após o descascamento, foi moído em moinho de discos com abertura de modo a se obter produto com granulometria semelhante ao grits de milho. Essa escolha baseou-se em procedimentos operacionais e experiências práticas de laboratório. A configuração do parafuso selecionada indicou que amostras com tamanhos de partículas maiores produziam extrudados mais leves e crocantes na extrusora comercial utilizada (Inbra RX50). Além disso, farinhas muito finas apresentam maior área de contato com a água levando a formação de grumos no interior do canhão, dificultando o processamento.

O maior percentual de retenção de partículas, tanto de feijão carioca quanto de grits de milho, foi verificado a $853 \mu \mathrm{m}$ (Figura 1). Partículas maiores também foram evidenciadas para essas matérias-primas, com tamanhos entre 1000 e $1200 \mu \mathrm{m}$. Observou-se que o grits de milho manteve maior homogeneidade quanto ao tamanho de partículas (entre 710 e $1200 \mu \mathrm{m}$ ). O feijão carioca apresentou tamanhos de partículas em todas as faixas analisadas, possivelmente devido ao tipo de moagem aplicado à essa matéria-prima, resultando em pequenas parcelas de farinha mais fina.

Ascheri e Carvalho (2008) consideram desejável, porém não necessariamente essencial, que as partículas das matérias-primas tenham tamanho e densidade uniformes para prevenir a segregação durante a mistura e o transporte antes da extrusão. A uniformidade no tamanho das partículas dos ingredientes permite que essas sejam adequada e igualmente cozidas no processo de extrusão, prevenindo dureza ou cozimento parcial do produto. Quando o tamanho das partículas da matéria-prima é muito variado, o produto final pode conter partículas indesejáveis com diferentes graus de cocção, o que diminui a qualidade da sua aparência e sabor.

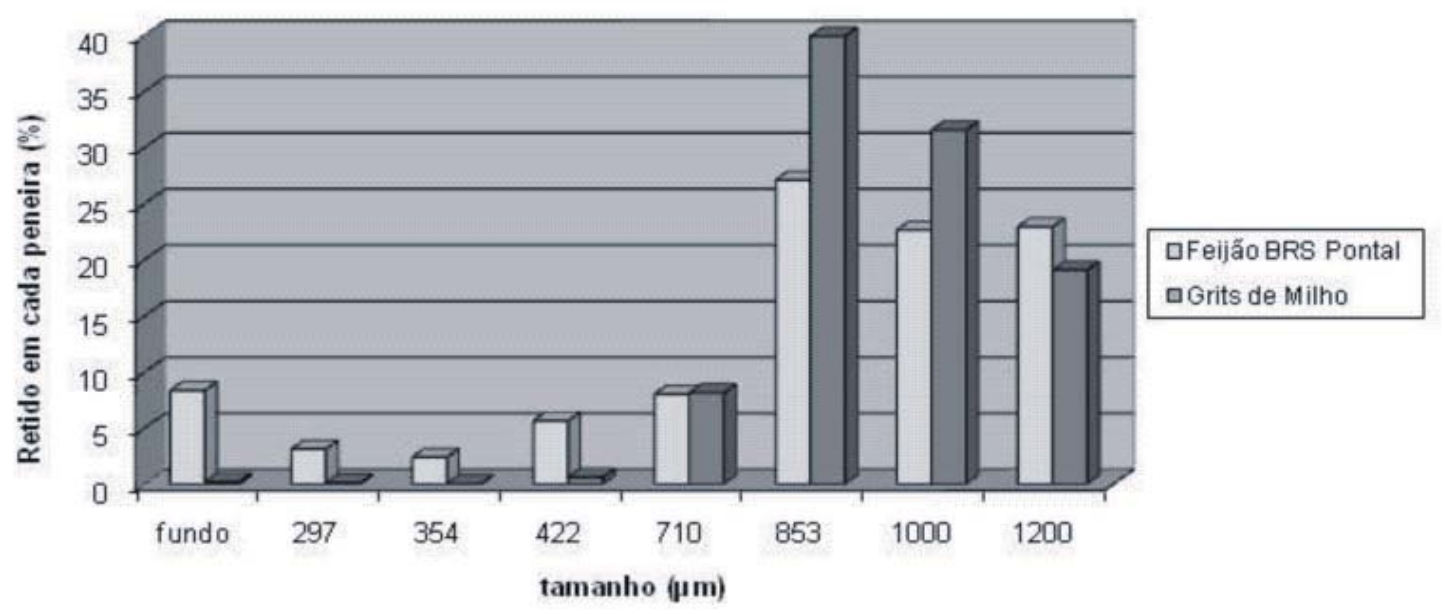

\section{FIGURA 1 - CLASSIFICAÇÃO GRANULOMÉTRICA DO FEIJÃO TIPO CARIOCA E GRITS DE MILHO}

\subsection{DELINEAMENTO EXERIMENTAL E CARACTERIZAÇÃO FÍSICA DAS FARINHAS MISTAS DE MILHO E FEIJÃO CARIOCA}

Por meio da Tabela 1 podem ser visualizados o delineamento experimental proposto, assim como os resultados de índice de solubilidade em água (ISA), índice de absorção de água (IAA) e as viscosidades inicial a $25^{\circ} \mathrm{C}(\mathrm{V} 25)$, máxima (VMax) e final (VF), tanto das farinhas mistas cruas quanto das pré-cozidas. 
TABELA 1 - ÍNDICE DE SOLUBILIDADE EM ÁGUA (ISA), ÍNDICE DE ABSORÇÃO DE ÁGUA (IAA) E VISCOSIDADE DE PASTA DAS FARINHAS PRÉ-COZIDAS E CRUAS DE MILHO E FEIJÃO CARIOCA

\begin{tabular}{|c|c|c|c|c|c|c|c|c|}
\hline Tratamentos & $x_{1}$ & $x_{2}$ & $x_{3}$ & ISA (\%) & $\begin{array}{l}\text { IAA } \\
(g / g)\end{array}$ & $\mathrm{V} 25(\mathrm{cP})$ & $\begin{array}{l}\text { VMax } \\
\text { (cP) }\end{array}$ & $\begin{array}{l}\text { VF } \\
\text { (cP) }\end{array}$ \\
\hline 1 & 333 & 13 & 15 & 11,84 & 4,60 & 490 & 38 & 120 \\
\hline 2 & 378 & 13 & 15 & 13,32 & 6,01 & 607 & 50 & 135 \\
\hline 3 & 333 & 19 & 15 & 15,20 & 6,18 & 701 & 117 & 278 \\
\hline 4 & 378 & 19 & 15 & 17,29 & 6,11 & 471 & 116 & 298 \\
\hline 5 & 333 & 13 & 45 & 11,93 & 5,50 & 628 & 126 & 306 \\
\hline 6 & 378 & 13 & 45 & 12,28 & 5,56 & 608 & 103 & 294 \\
\hline 7 & 333 & 19 & 45 & 9,60 & 5,86 & 162 & 262 & 535 \\
\hline 8 & 378 & 19 & 45 & 11,43 & 5,64 & 168 & 217 & 508 \\
\hline 9 & 355 & 16 & 30 & 12,56 & 6,03 & 636 & 127 & 316 \\
\hline 10 & 355 & 16 & 30 & 11,00 & 5,63 & 376 & 137 & 392 \\
\hline 11 & 355 & 16 & 30 & 13,60 & 6,05 & 395 & 101 & 266 \\
\hline 12 & 355 & 16 & 30 & 14,62 & 6,24 & 362 & 126 & 310 \\
\hline 13 & 355 & 16 & 30 & 13,57 & 6,09 & 354 & 142 & 390 \\
\hline 14 & 355 & 16 & 30 & 12,97 & 6,26 & 392 & 150 & 272 \\
\hline 15 & 318 & 16 & 30 & 13,77 & 5,99 & 321 & 162 & 275 \\
\hline 16 & 393 & 16 & 30 & 15,39 & 6,01 & 458 & 90 & 266 \\
\hline 17 & 355 & 10,9 & 30 & 13,24 & 5,90 & 687 & 99 & 217 \\
\hline 18 & 355 & 21,0 & 30 & 12,62 & 6,31 & 333 & 191 & 425 \\
\hline 19 & 355 & 16 & 4,8 & 20,64 & 5,86 & 524 & 65 & 161 \\
\hline 20 & 355 & 16 & 55,2 & 12,28 & 6,15 & 748 & 298 & 588 \\
\hline Milho (grits) & - & - & - & 1,80 & 2,60 & 5 & 1485 & 5384 \\
\hline Feijão BRS Pontal & - & - & - & 11,15 & 2,94 & 6 & 33 & 113 \\
\hline $\begin{array}{l}\text { Mistura crua_4,8\% } \\
\text { feijão }\end{array}$ & - & - & - & 1,96 & 2,63 & 5 & 1437 & 5120 \\
\hline $\begin{array}{l}\text { Mistura crua_15\% } \\
\text { feijão }\end{array}$ & - & - & - & 2,38 & 2,69 & 0 & 1010 & 3234 \\
\hline $\begin{array}{l}\text { Mistura crua_30\% } \\
\text { feijão }\end{array}$ & - & - & - & 3,96 & 2,78 & 3 & 574 & 1868 \\
\hline $\begin{array}{l}\text { Mistura crua_45\% } \\
\text { feijão }\end{array}$ & - & - & - & 6,52 & 2,78 & 0 & 123 & 1025 \\
\hline $\begin{array}{l}\text { Mistura crua_55,2\% } \\
\text { feijão }\end{array}$ & - & - & - & 6,04 & 2,83 & 0 & 65 & 957 \\
\hline
\end{tabular}

$\mathbf{X}_{1}=$ Rotação do parafuso (rpm); $\mathbf{X}_{2}=$ Umidade (\%); $\mathbf{X}_{3}=$ Feijão (\%); ISA = Índice de Solubilidade em Água (\%); IAA = Índice de Absorção de Água (g gel/g matéria seca); $\mathbf{V} 25$ = viscosidade máxima a $25^{\circ} \mathrm{C}$; VMax = viscosidade máxima a $95^{\circ} \mathrm{C}$; $\mathbf{V F}=$ viscosidade final.

\section{3 ÍNDICE DE SOLUBILIDADE EM ÁGUA (ISA)}

O índice de solubilidade em água (ISA) está relacionado à quantidade de sólidos solúveis presentes na amostra seca, permitindo verificar o grau de intensidade do tratamento térmico em função da gelatinização, dextrinização e consequente solubilização do amido (CARVALHO, 
ASCHERI e VIDAL, 2002). Esse índice é bastante utilizado para medir o grau de solubilização do amido extrudado em bebidas, sopas e alimentos infantis, entre outros. O ISA assume importância na caracterização de farinhas extrudadas para fins de solubilização posterior (caso de farinhas pré-gelatinizadas), pois permite verificar o grau de cozimento do amido e avaliar as condições de solubilização em meio aquoso. Para o preparo de alimentos reconstituídos torna-se necessário que a solubilização do material esteja de acordo com as características sensoriais desejadas.

O produto elaborado deve garantir homogeneidade com completa interação entre os componentes sólidos e a água. O ISA tem sido utilizado como indicador do grau de cozimento de extrudados de feijão (LAZOU e KROKIDA, 2010).

Geralmente, os valores de ISA para extrudados à base de milho giram em torno de 8,88 a $32,79 \%$. Os valores de ISA para a mistura de milho e feijão variaram entre 9,60 e 20,64 \% (Tabela 1), sendo similares aos verificados por Atienzo-Lazos et al. (2011), avaliando a mistura de feijão e milho nixtamalizado. Os valores de solubilidade nas amostras cruas foram notavelmente superiores para o feijão BRS pontal $(11,15 \%)$, quando comparado com o milho $(1,80 \%)$. Isso sugere que as proteínas presentes no feijão possam se solubilizar, quando não há tratamento térmico. Porém, após o processamento por extrusão houve agregação e interação entre os componentes dessa leguminosa favorecendo a redução desse índice.

Por meio da Tabela 2 e da Figura 2 podem ser observados os resultados obtidos após aplicação do teste $\mathrm{F}$ da análise de variância para o índice de solubilidade em água nas farinhas extrudadas de milho e feijão. Observou-se que apenas o conteúdo de feijão acrescentado ao milho foi significativo $(p<0,01)$, influenciando de forma linear e negativa os valores de ISA. Fato também verificado por Lazou e Krokida (2010) ao adicionarem lentilha à mistura com milho. Balandran-Quintero et al. (1998) constataram que extrudados com alto teor de proteínas apresentam menores valores de solubilidade em água. Além disso, nota-se influência significativa de forma negativa da interação entre a umidade e o feijão sobre a solubilidade em água. Isso quer dizer que maior teor de feijão, muitas vezes associado ao aumento do conteúdo de umidade, implica na redução dos valores de ISA.

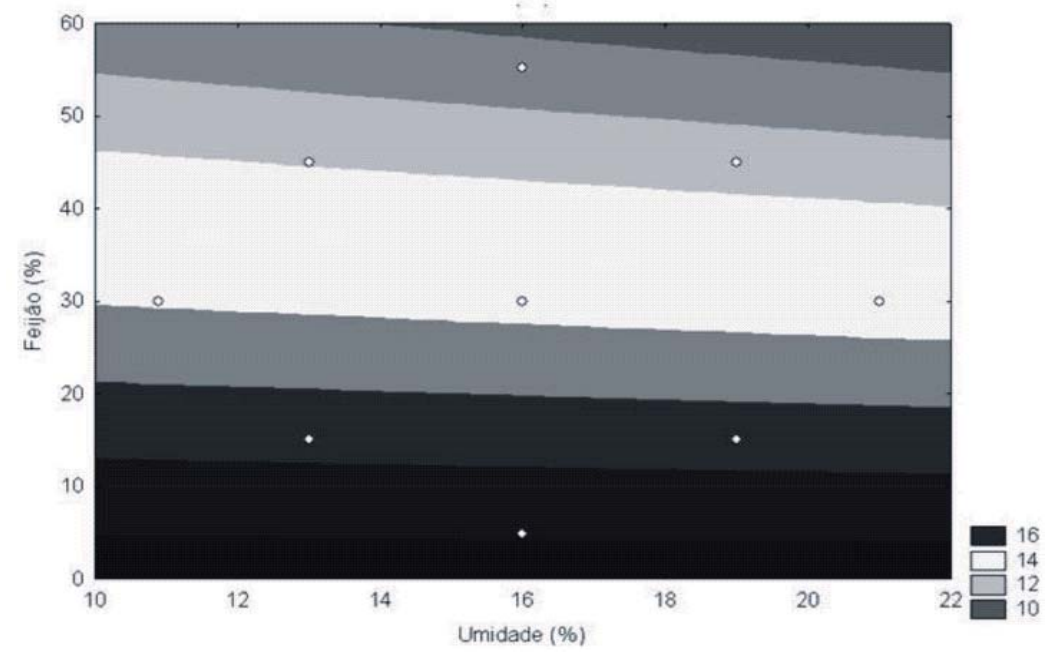

\section{FIGURA 2 - EFEITO DAS VARIÁVEIS QUANTITATIVAS UMIDADE (\%) E FEIJÃO (\%) NO ÍNDICE DE SOLUBILIDADE EM ÁGUA (ISA) DAS FARINHAS MISTAS EXTRUDADAS À BASE DE MILHO E FEIJÃO}

Lazou e Krokida (2010) verificaram que a solubilidade de extrudados elaborados com milho e lentilha foi significativamente afetada pela proporção de lentilha na mistura e pela umidade das amostras. Foi observada redução do ISA conforme aumento da umidade na mistura.

Não foi evidenciada relação entre a velocidade do parafuso e os valores de solubilidade, indicando que a máxima rotação aplicada no extrusor (393 rpm) não foi capaz de alterar significativamente a estrutura amilácea, degradando-a. Isso contradiz os achados de Ascheri et al. (2006), que observaram efeito positivo da rotação do parafuso sobre o ISA de farinhas mistas de arroz e bagaço de jabuticaba extrudadas. 
Mesa et al. (2009) ao fortificarem extrudados produzidos à base de milho e soja observaram que o ISA foi maior conforme o aumento da velocidade do parafuso (330 rpm), inclusive nas duas maiores concentrações de soja na mistura. Tal fato sugere que o aumento da velocidade do parafuso degradou as moléculas de amido em pequenos fragmentos, favorecendo sua solubilização em água.

A presença do feijão, associada à umidade durante o processo de extrusão, possivelmente forma rede proteica que impede em parte a perda de outras moléculas. Embora as proteínas de leguminosas apresentem sítios hidrofílicos, o processo de desnaturação que ocorre durante a extrusão limita a capacidade de hidratação dessas proteínas. Essa menor capacidade de hidratação é favorecida pela formação de pontes intra e intermoleculares das proteínas com as cadeias de amilose e amilopectina. O processo de extrusão, em alguns casos, favorece a reação de Maillard tendo como consequência a formação de agregado com maior massa molecular, contribuindo para a redução dos valores de ISA.

Por meio do coeficiente de determinação evidenciado na análise de variância pode-se verificar que $83 \%$ da variação total foi explicada pelo modelo aplicado. O erro experimental (17\%) pode estar associado a acuidade visual do analista em proceder a análise do ISA, que requer exatidão e padronização dos procedimentos. Também não foi evidenciada falta de ajuste dos dados desse experimento, sugerindo adequação do modelo de regressão quadrático aplicado.

\section{4 ÍNDICE DE ABSORÇÃO DE ÁGUA (IAA)}

O índice de absorção de água mostra a capacidade do grânulo de amido em absorver água, mesmo em temperatura ambiente e reflete a capacidade de integralidade da estrutura amilácea após o processo de extrusão.

A capacidade de absorção de água do material amiláceo cru torna-se geralmente elevada a partir do momento em que se aplica calor em meio úmido por meio do processo de gelatinização. Logo após o processo, o material extrudado é encaminhado para estufa para evaporação de parte da água livre que resulta em produto pré-cozido. $\mathrm{O}$ amido cru absorve apenas $30 \%$ do seu volume em água em temperatura ambiente e sem tratamento térmico. Essa capacidade de absorção elevase radicalmente conforme a temperatura do sistema aumenta até a gelatinização do amido ser atingida, quando haverá "quebra" na estrutura amilácea, fazendo com que o índice de absorção diminua (ASCHERI e CARVALHO, 2008).

Os valores de absorção para extrudados à base de milho normalmente giram em torno de 4,35 a $6,06 \mathrm{~g}$ gel/ $\mathrm{g}$ matéria seca. Para os extrudados de milho e feijão, os valores de IAA variaram entre 4,60 e 6,31 g gel/ g matéria seca (Tabela 1).

Por meio da Tabela 2 verifica-se que, após a aplicação do teste $F$ da análise de variância para o IAA em farinhas extrudadas de milho e feijão, não houve influência de nenhuma das variáveis quantitativas (rotação do parafuso, umidade e feijão) sobre a absorção de água das farinhas. $O$ mesmo efeito foi verificado por Atienzo-Lazos et al. (2011) em misturas de feijão e milho nixtamalizado e também por Altan, McCarthy e Maskan (2008) no desenvolvimento de extrudados à base de cevada. No entanto, Carvalho et al. (2010) observaram que o tamanho de partículas do fubá utilizado influenciou significativamente de forma negativa os valores de absorção de água das farinhas extrudadas. As partículas menores apresentam maior área de contato durante o processamento, 0 que pode levar ao aumento do cisalhamento até certo ponto, elevando a capacidade de absorção de água. Mesa et al. (2009) verificaram menor IAA em misturas de milho e soja conforme o aumento da velocidade do parafuso.

Alguns fatores podem afetar os valores de IAA, além da temperatura. A taxa de cisalhamento pode provocar ruptura da estrutura amilácea e diminuir os valores desse índice, conforme afirmaram Hagenimana, Ding e Fang (2006). Além disso, a retrogradação que pode ocorrer durante a estocagem do material diminui a quantidade de amido disponível para absorver água. Lazou e Krokida (2010) observaram que o IAA de extrudados de lentilha e milho foi influenciado significativamente pela proporção de lentilha na mistura e pela umidade na massa. 
De acordo com os resultados obtidos do presente estudo, a adição de feijão em concentrações de até 55 \% não afetou significativamente a absorção de água do material extrudado. O mesmo ocorreu para os teores de umidade utilizados (11 a $21 \%$ ), assim como a rotação do parafuso (318 a $393 \mathrm{rpm}$ ).

Para a reconstituição de farinhas pré-gelatinizadas é desejável que parte da estrutura amilácea se mantenha íntegra, pois ao acrescentar água ocorre o espessamento do produto que reduz a necessidade de aditivos (como espessantes).

Certos cuidados devem ser tomados ao afirmar que nenhuma das variáveis quantitativas exerceram efeito sobre a solubilidade, pois apenas $62 \%$ da variação total foi explicada pelo modelo, atribuindo-se em torno de $40 \%$ aos resíduos. Cabe ressaltar que os erros mais comuns durante essa avaliação estão relacionados a acuidade visual do analista e padronização dos procedimentos de análise. Mesmo assim, não foi evidenciada falta de ajuste dos dados em relação ao modelo quadrático aplicado. Portanto, por mais que o coeficiente de determinação tenha sido baixo, podese considerar que o modelo aplicado é preditivo para a variável resposta em questão.

\subsection{VISCOSIDADE DAS FARINHAS EXTRUDADAS}

A viscosidade de pasta depende da avaliação do grau de gelatinização do amido e da taxa de fragmentação e solubilização molecular.

As amostras extrudadas apresentam maior viscosidade inicial a $25^{\circ} \mathrm{C}$ quando comparadas com as misturas cruas (Tabela 1). Isto significa que as amostras extrudadas apresentam boas características de viscosidade quando acrescidas em meio aquoso à temperatura ambiente. Como esperado, o valor de viscosidade máxima a $95^{\circ} \mathrm{C}$ da amostra extrudada mostrou-se inferior ao da mistura crua. É importante para o preparo de sopas, que essas não alcancem valores elevados para viscosidade final no ciclo de resfriamento, pois esse produto deve apresentar boa cremosidade, homogeneidade e textura, mesmo após o seu preparo em temperaturas mais amenas.

Carvalho et al. (2010) observaram valores elevados da viscosidade máxima a $25^{\circ} \mathrm{C}(\mathrm{V} 25)$ em farinhas extrudadas à base de grits de milho. Sugeriram que o aumento da taxa de cisalhamento durante a extrusão pode levar as moléculas a apresentarem mais sítios hidrofílicos expostos a ligações com a água, formando estrutura tri-dimensional quando em temperatura ambiente. Não houve evidência de picos de viscosidade a $95^{\circ} \mathrm{C}$, o que é típico para materiais que foram submetidos ao processo de extrusão (amidos pré-gelatinizados). O tamanho das partículas das matérias-primas não influenciou os valores de viscosidade máxima a $95^{\circ} \mathrm{C}$. Esse tipo de viscosidade indica o início do período de resfriamento em que materiais ainda quentes começam a ser resfriados, dando início a re-associação das cadeias de amilose e a consequente retrogradação.

\subsubsection{Viscosidade máxima inicial a $25^{\circ} \mathrm{C}(\mathrm{V} 25)$}

A viscosidade inicial, também conhecida por viscosidade de pasta a frio, indica a capacidade do alimento extrudado em absorver água em temperatura ambiente e formar pasta, gel ou líquido viscoso (FENIMAN, 2004).

Por meio da Tabela 2 verifica-se que apenas a rotação do parafuso, assim como a interação entre a rotação e o conteúdo de feijão não influenciaram os valores da V25. Foram observados efeitos lineares e quadráticos das variáveis dependentes sobre a viscosidade máxima a $25^{\circ} \mathrm{C}$. A umidade inicial nas misturas de milho e feijão exerceu efeito significativo $(p<0,01)$ e negativo nos valores de $\mathrm{V} 25$. O aumento da umidade no material exerce efeito lubrificante no interior do canhão, que tende a reduzir sua temperatura interna, ocorrendo menor cisalhamento das amostras e menor cocção do material. Isso faz com que a estrutura amilácea não apresente boa capacidade para absorver água em temperatura ambiente.

A adição de feijão influenciou significativamente $(p<0,05)$ e de forma negativa os valores de V25. O acréscimo de material com considerável quantidade de proteínas e fibras, do tipo solúvel (no endosperma), reduz a quantidade total de amido na mistura. 
Foi observado que as interações entre rotação e umidade, assim como umidade e feijão influenciaram de forma negativa os valores de V25. Efeitos similares foram verificados por Silva (2006) ao avaliar os parâmetros de extrusão na viscosidade a $25^{\circ} \mathrm{C}$ de extrudados de arroz e pó de café, tendo a presença do café na mistura sido a variável com maior contribuição para a redução dos valores de viscosidade a frio.

A substituição de parte da concentração de amido do material a ser extrudado exige maior temperatura para a formação da V25. Tanto as fibras quanto as proteínas não fornecem viscosidade suficiente ao meio, diminuindo a gelatinização do amido (ASCHERI et al., 2006).

Nota-se que $77 \%$ da variação total foi explicada pelo modelo, atribuindo-se $23 \%$ aos resíduos. Além do valor referente ao erro experimental ser considerado elevado, houve falta de ajuste dos dados ao modelo quadrático aplicado. No entanto, não há sugestão de utilização de outro modelo em razão do seu melhor ajuste aos demais experimentos.

Por meio das Figuras 3 e 4 verifica-se o efeito das variáveis quantitativas umidade e feijão, assim como da umidade e rotação, respectivamente, nos valores de V25. Os valores dessa variável oscilaram entre 162 e 748 cP (Tabela 1).

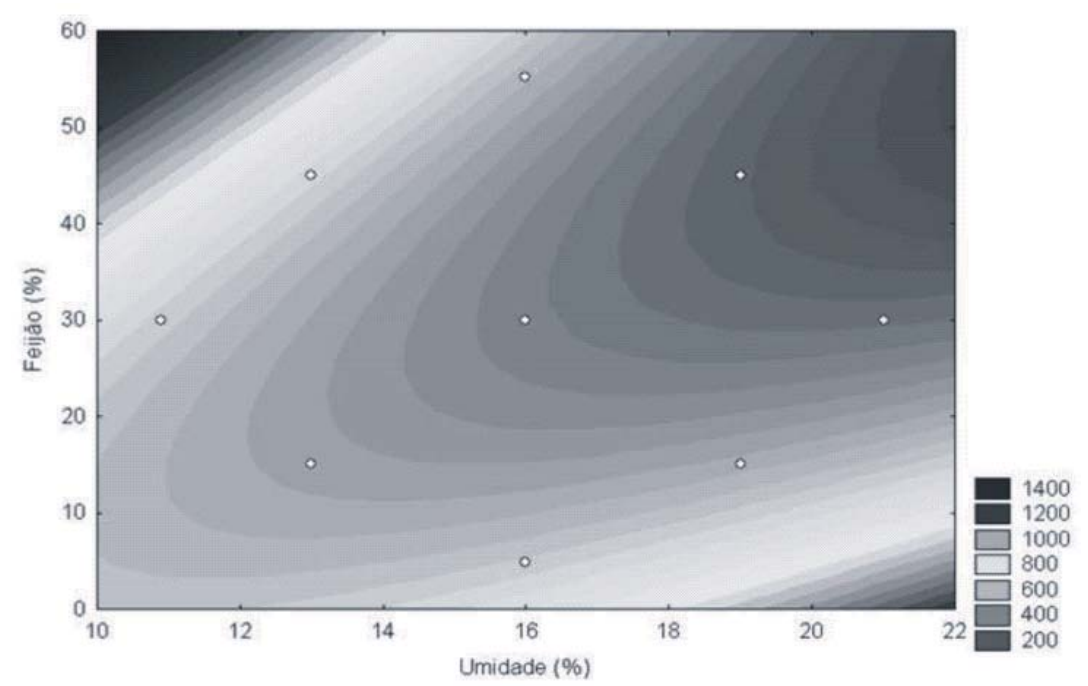

FIGURA 3 - EFEITO DAS VARIÁVEIS QUANTITATIVAS FEIJÃO (\%) E UMIDADE (\%) NA VISCOSIDADE MÁXIMA A $25^{\circ} \mathrm{C}$ (V25) DAS FARINHAS MISTAS EXTRUDADAS À BASE DE MILHO E FEIJÃO

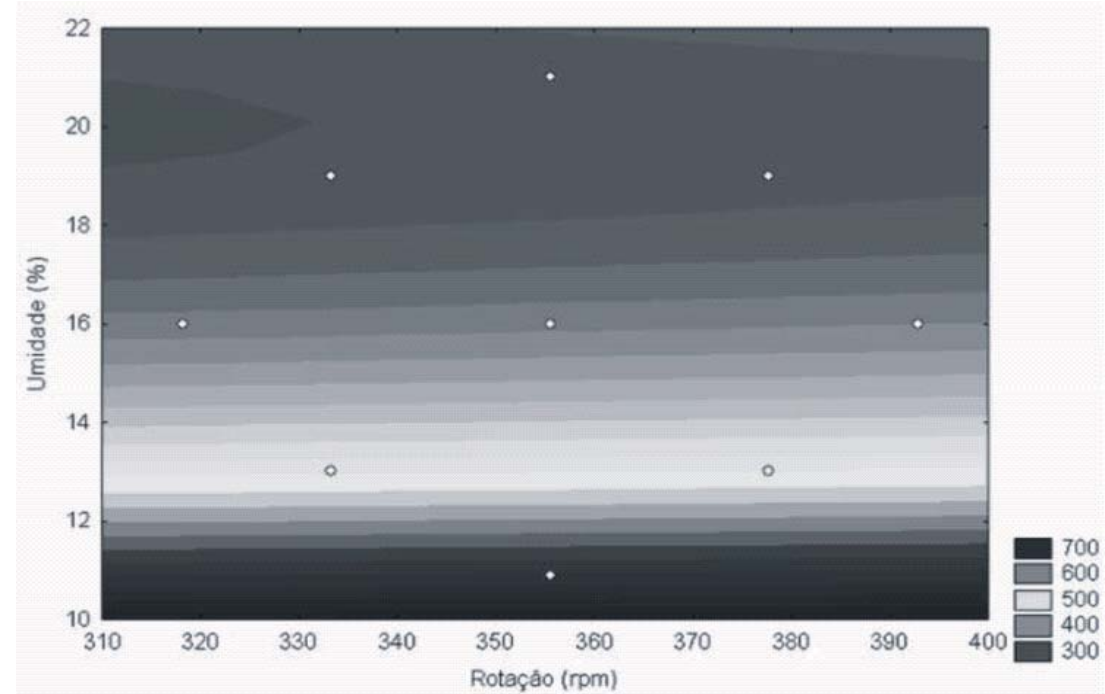

FIGURA 4 - EFEITO DAS VARIÁVEIS QUANTITATIVAS UMIDADE (\%) E ROTAÇÃO (RPM) NA VISCOSIDADE MÁXIMA A $25^{\circ} \mathrm{C}$ (V25) DAS FARINHAS MISTAS EXTRUDADAS À BASE DE MILHO E FEIJÃO 


\subsubsection{Viscosidade máxima a $95^{\circ} \mathrm{C}(\mathrm{VMax})$}

A viscosidade máxima a $95^{\circ} \mathrm{C}$ (VMax) caracteriza-se pelas transformações que ocorrem na estrutura granular do amido submetido ao processo de aquecimento, como resultado da desorganização molecular, que leva ao entumescimento total e gelatinização com absorção máxima de água pelos grânulos de amido. Valores de VMax elevados geralmente são verificados quando o processamento aplicado não é suficiente para despolimerizar a estrutura amilácea, favorecendo a dextrinização do material. Assim, grânulos de amido intactos chegam ao sistema quando sofrem gelatinização na fase de aquecimento. A viscosidade máxima no ciclo de aquecimento indica as possibilidades de utilização da farinha extrudada. Torna-se importante quando, por exemplo, a farinha for destinada ao preparo de sopas, tortas ou outros alimentos em que seja necessário conservar a viscosidade em temperatura acima da ambiente (TORRES et al., 2005).

Tanto a umidade quanto o conteúdo de feijão foram significativos $(p<0,01)$, influenciando linearmente de forma positiva os valores da viscosidade máxima a $95^{\circ} \mathrm{C}$ (Tabela 2). Como citado anteriormente, tanto o conteúdo de umidade quanto o de feijão exerceram efeito negativo na V25 fazendo com que a estrutura amilácea sofresse menor cocção e degradação da molécula. Com isso, o amido que não sofreu cocção completa assim como despolimerização e desarranjo molecular de forma a interagir com moléculas de água chega ao ciclo de aquecimento de forma intacta. Seria durante esse ciclo que as estruturas amiláceas que não sofreram alterações iniciariam o processo de gelatinização no sistema viscoamilográfico aumentando os valores de viscosidade a $95^{\circ} \mathrm{C}$.

Silva (2006) verificou que a presença de outra matéria-prima, não amilácea, reduziu de forma significativa a viscosidade máxima a $95^{\circ} \mathrm{C}$. O mesmo foi constatado por Borges (2002) trabalhando com farinhas de arroz e quinoa, por Mendonça (2005) estudando farinha de arroz e amaranto integral e por Ascheri et al. (2006) avaliando farinha de arroz e casca de jabuticaba.

Verificou-se que $92 \%$ da variação total foi explicada pelo modelo, não sendo evidenciada falta de ajuste dos dados ao modelo quadrático aplicado, sugerindo que esse seja preditivo à análise em questão. Notou-se também que o intercepto foi significativo $(p<0,01)$, indicando escolha adequada do ponto central. Por meio da Figura 5 verifica-se o efeito das variáveis quantitativas umidade e feijão nos valores de VMax, que oscilaram entre 38 e 298 cP (Tabela 1). O valor mínimo atingido está relacionado ao tratamento 1 (333 rpm; 13\% umidade e 15\% feijão) e o maior ao tratamento 20 (356 rpm; 16\% umidade e 55,2\% feijão). Ray e Mishra (2006) afirmaram que os grânulos de amido provenientes do milho exigem maior temperatura para iniciar o processo de gelatinização devido ao elevado grau de cristalinidade da sua estrutura molecular.

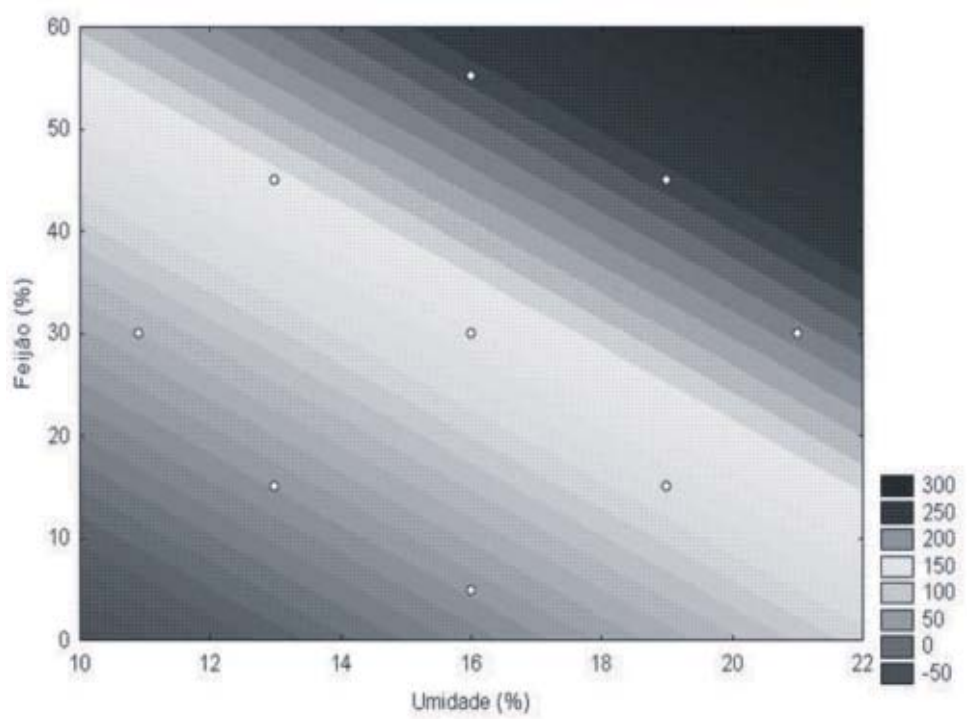

FIGURA 5 - EFEITO DAS VARIÁVEIS QUANTITATIVAS FEIJÃO (\%) E UMIDADE (\%) NA VISCOSIDADE MÁXIMA A $95^{\circ} \mathrm{C}$ (VMAX) DAS FARINHAS MISTAS EXTRUDADAS À BASE DE MILHO E FEIJÃO 


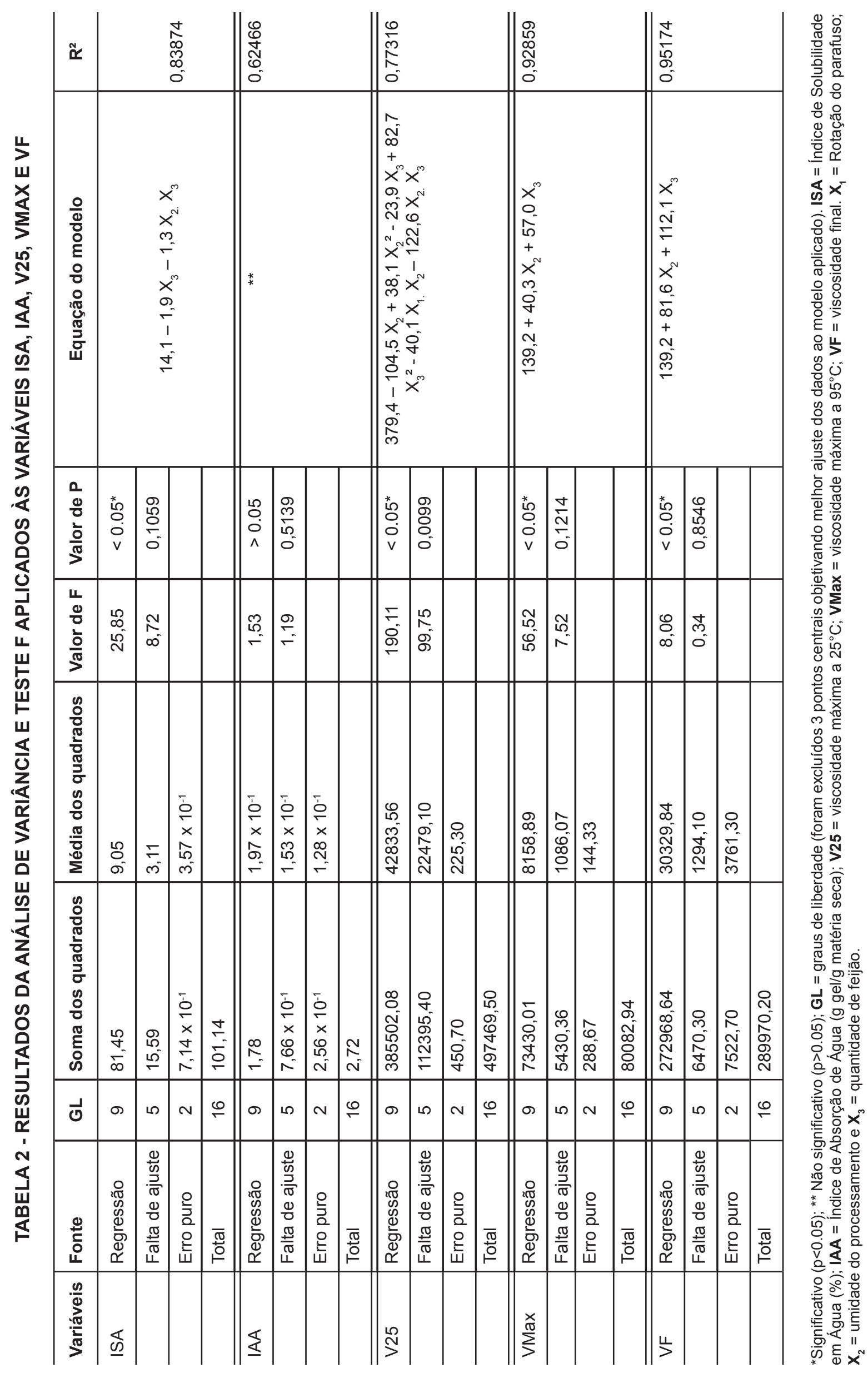




\subsubsection{Viscosidade final (VF)}

Após o aquecimento, gelatinização e resfriamento dos grânulos de amido, uma das características importantes em alimentos. (principalmente durante o armazenamento) é o processo de retrogradação. Esse fenômeno ocorre devido ao efeito da recristalização das moléculas de amilose e amilopectina, culminando na reorganização da estrutura molecular e, consequentemente, no aumento da viscosidade durante o processo de resfriamento (SILVA, 2006).

Apenas as variáveis lineares de umidade e feijão influenciaram significativamente $(p<0,05)$, de forma positiva, os valores de VF (Tabela 2). Pode-se sugerir que as amostras contendo maiores teores de umidade, assim como de feijão, foram mais susceptíveis à retrogradação. $O$ amido que sofreu gelatinização e que não teve sua estrutura degradada passa agora pelo ciclo de resfriamento em que as cadeias de amilose tendem a reorganização, ocorrendo sinérese e posterior retrogradação. Durante a avaliação das características de viscosidade de sopas elaboradas com feno-grego, Ravindran e Matia-Merino (2009) evidenciaram que a viscosidade final dessas amostras aumentou conforme a elevação do teor de feno-grego. A viscosidade final dessas sopas foi significativamente maior quando comparada com sopas elaboradas apenas com amido de milho. Carvalho, Ascheri e Vidal (2002) verificaram que a tendência a retrogradação em extrudados com farinha de arroz, banana e trigo diminui com a redução da umidade. Isto possivelmente está relacionado à maior taxa de cisalhamento conferida no momento da extrusão, o que reduz a capacidade de reorganização das cadeias de amilose devido a despolimerização e rearranjo da estrutura amilácea.

Notou-se que 95\% da variação total foi explicada pelo modelo, não sendo evidenciada falta de ajuste dos dados ao modelo quadrático aplicado, sugerindo sua adequação para a análise dos valores de viscosidade final.

Por meio da Figura 6 verifica-se o efeito das variáveis quantitativas umidade e feijão nos valores de VF, que oscilaram entre 120 e $588 \mathrm{cP}$ (Tabela 1). O valor mínimo atingido está relacionado ao tratamento 1 (333 rpm; 13\% umidade e 15\% feijão) e o maior ao tratamento 20 (356 rpm; 16\% umidade e $55,2 \%$ feijão), assim como observado nos resultados de VMax.

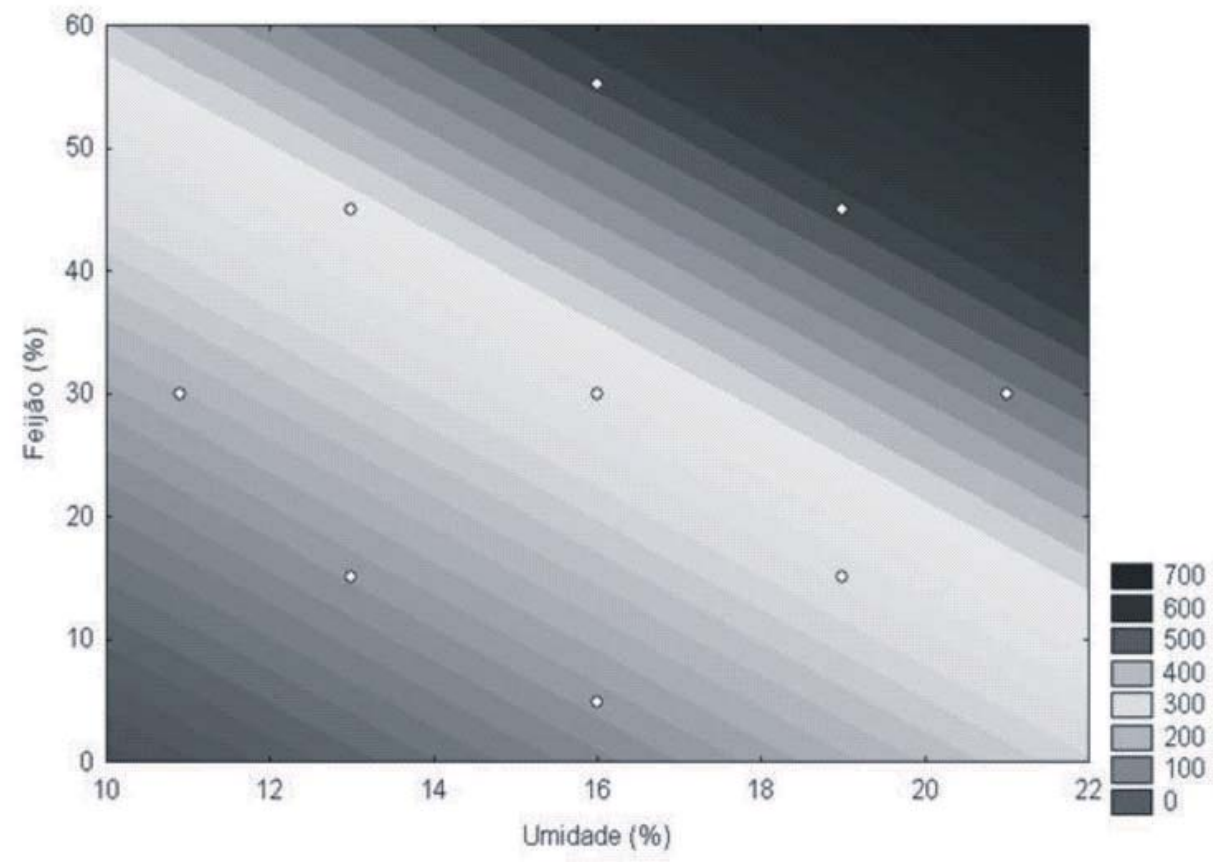

FIGURA 6 - EFEITO DAS VARIÁVEIS QUANTITATIVAS FEIJÃO (\%) E UMIDADE (\%) NA VISCOSIDADE FINAL (VF) DAS FARINHAS MISTAS EXTRUDADAS À BASE DE MILHO E FEIJÃO 
Para o preparo de sopas instantâneas, valores de solubilidade em água elevados podem indicar que o material extrudado sofreu dextrinização, desestruturando a cadeia amilácea. É importante que boa parte da estrutura amilácea permaneça intacta de forma que seja capaz de absorver água no momento da reconstituição para apresentar a viscosidade e a textura desejáveis. Além disso, o preparo de sopas instantâneas não exige que a água adicionada esteja em temperatura de ebulição. O ideal é acrescentar água potável em temperatura suportável pelo paladar. Após o preparo das sopas, durante seu resfriamento, o material não deve sofrer retrogradação imediata para que não apresente aspecto endurecido. Dessa forma, para a elaboração de farinhas pré-gelatinizadas com características desejáveis para o preparo de sopas foram considerados os seguintes limites para cada variável resposta estudada: ISA, 12-16\%; IAA, 5,6-6,2 g gel/g matéria seca; V25, 300100 cP; VMax, 100-160 cP e VF 200-400 cP. Esses limites foram observados por meio de correlações entre a variável ISA e as demais variáveis resposta, considerando-se o valor de ISA intermediário desejável para o preparo de sopas.

Aplicando-se a função de desejabilidade para o ponto ótimo foram obtidos os valores desejáveis para cada variável analisada (Tabela 3).

\section{TABELA 3 - PONTOS ÓTIMOS CONSIDERADOS PARA A ELABORAÇÃO DE FARINHAS PRÉ-GELATINIZADAS}

\begin{tabular}{l|c|l|c}
\hline \multicolumn{2}{c|}{ VARIÁVEIS INDEPENDENTES } & \multicolumn{2}{c}{ VARIÁVEIS RESPOSTA } \\
\hline Rotação (rpm) & 380,5 & ISA (\%) & 13,07 \\
\hline Umidade (\%) & 15,9 & IAA (g gel/g m.s.) & 5,81 \\
\hline Feijão (\%) & 42,6 & V25 (cP) & 436,52 \\
\hline & & VMax (cP) & 157,86 \\
\hline & & VF (cP) & 390,26 \\
\hline
\end{tabular}

O valor da desejabilidade foi de 0,903003 , considerado muito bom para o estudo em questão.

\section{CONCLUSÃO}

Por meio do estudo realizado verificou-se a possibilidade de obtenção de farinhas prégelatinizadas para o preparo de sopas de milho e feijão BRS Pontal tipo Carioca. Os parâmetros utilizados no processamento por extrusão termoplástica foram: rotação do parafuso $(318,2$ a 392,9 rpm); umidade inicial na mistura (10,9 a 21\%) e adição de feijão sem casca BRS Pontal do tipo Carioca $(4,8$ a $55,2 \%)$.

O aumento do conteúdo de feijão nas amostras reduziu a solubilidade das farinhas extrudadas, entretanto não afetou de forma significativa os valores de absorção em água.

A viscosidade de pasta apresentada pelas farinhas está condizente com a característica de fácil reconstituição, sendo solubilizadas sem a necessidade de cozimento. As farinhas prégelatinizadas de milho e feijão apresentaram-se viscosas, homogêneas e com pouca tendência a retrogradação. Isso quer dizer que o processo de extrusão e os parâmetros aplicados permitiram elaborar produtos com características desejáveis para o preparo de sopas, por exemplo. 


\section{ABSTRACT \\ EFFECT OF THERMOPLASTIC EXTRUSION ON THE CHARACTERISTICS OF PASTA VISCOSITY, SOLUBILITY AND WATER ABSORPTION OF PRE-COOKED FLOURS MADE WITH MAIZE AND CARIOCA TYPE BEANS (BRS PONTAL)}

The aim of this study was to evaluate the effect of the thermoplastic extrusion on pasta viscosity characteristics, solubility, water absorption of pregelatinized flours with maize and carioca type beans (BRS Pontal). The parameters used in this process were: screw speed (318.2 to $392.9 \mathrm{rpm})$, initial moisture (10.9 to $21 \%)$ and the addition of peeled carioca type beans (4.8 to 55.2\%). These parameters were set in levels, by a second order experimental composite rotational design where the response variables studied were water solubility index (WSI), water absorption index (WAI), initial viscosity (V25), maximum viscosity at $95^{\circ} \mathrm{C}(\mathrm{VMax})$ and final viscosity (FV). In the present study it was observed that higher levels of beans content increased the solubility of extruded flour, but did not affect significantly the values of water absorption. The flours viscosity profile is consistent with the characteristic of semi-instant products, being dissolved without needing to cook. The optimal points considered to develop pre-gel flours were: WSI (13.07\%); WAI (5.81 g gel/g dry matter); V25 (436.52 cP); VMax $(157.86 \mathrm{cP})$ and VF $(390.26 \mathrm{cP})$, to a value of desirability of 0.90 .

KEY-WORDS: EXTRUSION; PREGELATINIZED FLOURS; CORN; BEANS.

\section{REFERÊNCIAS}

1 ALTAN, A.; McCARTHY, K.L.; MASKAN, M. Evaluation of snack foods from barley-tomato pomace blends by extrusion processing. Journal of Food Engineering, v.84, p. 231-242, 2008.

2 ANDERSON, R. A.; CONWAY, H. F.; PFEIFER, V. F.; GRIFFIN JUNIOR, L. Gelatinization of corn grits by roll-and extrusion-cooking. Cereal Science Today, St. Paul, v.14, n.1, p. 4-12, 1969.

3 ANDRIGUETTO, J.M. Nutrição animal. As bases e os fundamentos da nutrição animal: os alimentos. São Paulo: Nobel, 2006. 389 p.

4 ASCHERI, D.P.R.; ANDRADE, C.T.; CARVALHO, C.W.P.; ASCHERI, J.L.R. Obtenção de farinhas mistas pré-gelatinizadas a partir de arroz e bagaço de jabuticaba: efeito das variáveis de extrusão nas propriedades de pasta. Boletim do CEPPA, Curitiba, v.24, n.1, p. 115-144, jan./jun. 2006.

5 ASCHERI, J.L.R.; CARVALHO, C.W.P. Curso de processo de extrusão de alimentos: aspectos tecnológicos para o desenvolvimento e produção de alimentos para consumo humano e animal. Rio de Janeiro: EMBRAPA, 2008. 81 p.

6 ATIENZO-LAZOS, M.; DELGADO, E.; OCHOA-MARTÍNEZ, A.; AGUILAR-PALAZUELOS, E.; MARTINEZ, B.F.; RAMIREZWONG, B.; GALLEGOS-INFANTE, J.A.; MEDRANO-ROLDAN, H.; SOLIS-SOTO, A. Effect of moisture and temperature on the functional properties of composite flour extrudates from beans (Phaseolus vulgaris) and nixtamalized corn (Zea mays). Journal of Animal Production Advances, v.1, n.1, p. 9-20, 2011.

7 BALANDRAN-QUINTERO, R.R.; BARBOSA-CANOVAS, G.V.; ZAZUETA-MORALES, J.J.; ANZALDUA-MORALES, A.; QUINTERO-RAMOS, A. Functional and nutritional properties of extruded whole pinto bean meal (Phaseolus vulgaris L.). Journal of Food Science, n. 36, p. 113-116, 1998.

8 BECKER, A.; HILL, S.E.; MITCHELL, J.R. Milling - a further parameter affecting the rapid visco analyser (rva) profile. Cereal Chemistry, n.78, p.166-172, 2001.

9 BORGES, J.T.S. Elaboração de macarrão pré-cozido à base de farinha integral de quinoa e farinha de arroz utilizando o processo de extrusão termoplástica. 2002. 116 f. Tese (Mestrado em Ciência e Tecnologia de Alimentos), Universidade Federal Rural do Rio de Janeiro, Rio de Janeiro, 2002.

10 BOX, G.E.P.; HUNTER, W.G.; HUNTER, J.S. Statistics for experimenters: an introduction to design, data analysis and model building. New York: John Wiley \& Sons, 1978.

11 CARVALHO, C.W.P.; TAKEITI, C.Y.; ONWULATA, C.I.; PORDESIMO, L.O. Relative effect of particle size on the physical properties of corn meal extrudates: effect of particle size on the extrusion of corn meal. Journal of Food Engineering, $n$. 98, p. 103-109, 2010.

12 CARVALHO, R.V.; ASCHERI, J.L.R.; VIDAL, J. Efeito dos parâmetros de extrusão nas propriedades físicas de pellets (3G) de misturas de farinhas de trigo, arroz e banana. Ciênc Agrotec., Lavras, v.26, n.5, p.1006-18, 2002.

13 CLERICI, M.T.P.S.; EL-DASH, A.A. Características tecnológicas de farinhas de arroz pré-gelatinizadas obtidas por extrusão termoplástica. Ciênc. Agrotec., Lavras, v.32, n.5, p. 1543-1550, set./out. 2008.

14 DERRINGER G.C.; SUICH, R. Simultaneous optimization of several responses variables. J. Quality Tech., v. 12, n. 4, p. 214-219, 1980. 
15 FENIMAN, C.M. Caracterização de raízes de mandioca do cultivar IAC-70 quanto à cocção, composição química e propriedades do amido em duas épocas de colheita. 2004. 83 f. Tese (Mestrado em Ciências), Escola Superior de Agricultura Luiz de Queiroz, Universidade de São Paulo, Piracicaba, 2004.

16 GOMES, J.C.; SILVA, C.O. Elaboração e análises físico-químicas de farinhas de feijão. Revista Ceres, v.50, n. 292, p. 687-697, 2003

17 GOMES, J.C.; SILVA, C.O.; COSTA, N.M.B.; PIROZI, M.R. Desenvolvimento e caracterização de farinhas de feijão. Revista Ceres, v.53, p. 548-558, 2006.

18 HAGENIMANA, A.; DING, X.; FANG, T. Evaluation of rice flour modified by extrusion cooking. Journal of Cereal Science, n.43, p. 38-46, 2006.

19 LAZOU, A.; KROKIDA, M. Functional properties of corn and corn-lentil extrudates. Food Research International, n.43, p. $609-616,2010$

20 MENDONÇA, X.M.F.D. A extrusão termoplástica no desenvolvimento de produtos derivados de amaranto. 2005. 182 f. Tese (Doutorado em Tecnologia de Processos Químicos e Bioquímicos) - Universidade Federal do Rio de Janeiro, Rio de Janeiro, 2005.

21 MESA, N.J.E.; ALAVI, S.; SINGH, N.; SHI, Y.; DOGAN, H.; SANG, Y. Soy protein-fortified expanded extrudates: baseline study using normal corn starch. Journal of Food Engineering, n.90, p. 262-270, 2009.

22 MESQUITA, F.R.; CORRÊA, A.D.; ABREU, C.M.P. Linhagens de feijão (Phaseolus vulgaris L.): composição química e digestibilidade protéica. Ciênc. Agrotec., Lavras, v. 31, n. 4, p. 1114-1121, jul./ago. 2007.

23 MIRANDA, R.A.; DUARTE, J.O.; GARCIA, J.C. Cultivo do milho. Mercado e comercialização. Sistema de produção, 1, versão eletrônica, Centro Nacional de Pesquisas em Milho e Sorgo (CNPMS), EMBRAPA, 8. ed. Outubro, 2012. Disponível em: <http://sistemasdeproducao.cnptia.embrapa.br>. Acesso em: 09 de maio de 2013.

24 RAVINDRAN, G.; MATIA-MERINO, L. Starch-fenugreek polysaccharide interactions in pure and soup systems. Food Hydrocolloids, n.23, p. 1047-1053, 2009.

25 RAY, T.; MISHRA, S. Morphology and functional properties of corn, potato and tapioca starches. Food Hydrocolloids, n.20, p. 557-566, 2006.

26 SILVA, R.F. Extrusão termoplástica de farinha de arroz com café torrado e moído e sua utilização em produtos de panificação. 2006. 116 f. Tese (Doutorado em Ciência dos Alimentos), Universidade de Lavras, Lavras, 2006.

27 STATISTICA software. V. 6.0. Tuksa, OK: Statsoft INC., 2001. CD-ROM.

28 TORRES, L.L.G.; EL-DASH, A.A.; CARVALHO, C.W.P.; ASCHERI, J.L.R.; GERMANI, R.; MIGUEZ, M. Efeito da umidade e da temperatura no processamento de farinha de banana verde por extrusão termoplástica. Boletim do CEPPA, Curitiba, v.23, n.2, p. 273-290, 2005.

\section{AGRADECIMENTOS}

À Empresa Brasileira de Pesquisa Agropecuária - Embrapa Agroindústria de Alimentos pelas instalações e equipamentos. Aos programas de biofortificação HarvestPlus, AgroSalud e BioFort. À Embrapa Arroz e Feijão pelos grãos de feijão cedidos. À Coordenação de Aperfeiçoamento de Pessoal de Nível Superior (Capes) pela bolsa de doutorado e ao Programa de Pós-Graduação em Ciência e Tecnologia de Alimentos da Universidade Federal Rural do Rio de Janeiro (UFRRJ). 